H.-H. Kuo, J. Potthoff and L. Streit

Nagoya Math. J.

Vol. 121 (1991), 185-194

\title{
A CHARACTERIZATION OF WHITE NOISE TEST FUNCTIONALS
}

\section{H.-H. KUO, J. POTTHOFF AND L. STREIT}

\section{§1. Introduction and main result}

In a recent paper [PS 89], two of the present authors have found a characterization of a certain space $(\mathscr{S})^{*}$ of generalized functionals of white noise, i.e. generalized functionals on $\mathscr{S}^{\prime}(\mathbb{R})$ equipped with the $\sigma$-algebra $\mathscr{B}$ generated by its cyclinder sets and with the white noise measure $\mu$ given by

$$
\int_{\mathscr{C}^{\prime}(\mathrm{R})} \exp (i\langle x, \xi\rangle) d \mu(x)=\exp \left(-\frac{1}{2}|\xi|_{2}^{2}\right)
$$

for $\xi \in \mathscr{S}(\mathbb{R})$. Here, $|\cdot|_{2}$ denotes the norm of $L^{2}(\mathbb{R})$, and $\langle\cdot, \cdot\rangle$ dual pairing. Below, we shall shortly recall the construction of the space $(\mathscr{S})^{*}$ as the dual of a space $(\mathscr{S})$ of "smooth" functionals on $\mathscr{S}^{\prime}(\mathbb{R})$. The characterization mentioned above is of considerable power: it provides an extremely convenient way to decide whether a certain given functional is an element in $(\mathscr{S})^{*}$. This has been shown in [PS 89] for a number of examples (especially for certain measures on $\mathscr{S}^{\prime}(\mathbb{R})$ ). The purpose of the present note is to give a similar characterization for the elements $(\mathscr{P})$ of test functionals. For notation, definitions, more background and references, we refer the reader to [PS 89].

Let $\Gamma(A)$ denote the second quantization of the self-adjoint $L^{2}(\mathbb{R})$ operator $A$ which on $\mathscr{S}(\mathbb{R})$ is defined as

$$
A \xi(u)=-\xi^{\prime \prime}(u)+\left(1+u^{2}\right) \xi(u), \quad \xi \in \mathscr{S}(\mathbb{R}), u \in \mathbb{R} .
$$

Let $\mathscr{P}$ denote the algebra of smooth polynomials on $\mathscr{S}^{\prime}(\mathbb{R})$, i.e. $\mathscr{P}$ is generated by the random variables $X_{\xi}=\langle\cdot, \xi\rangle, \xi \in \mathscr{S}(\mathbb{R})$. For $p \geq 0$, let $\mathscr{S}_{p}(\mathbb{R})$ denote the completion of $\mathscr{S}(\mathbb{R})$ with respect to the norm $|\xi|_{2, p}=$ $\left|A^{p} \xi\right|_{2}$, and let $(\mathscr{S})_{p}$ denote the completion of $\mathscr{P}$ with respect to the norm

Received May 17, 1990. 
$\|\varphi\|_{2, p}=\left\|\Gamma(A)^{p} \varphi\right\|_{2}$, where $\|\cdot\|_{q}, q \geq 1$, denotes the norm of $L^{q}\left(\mathscr{S}^{\prime}(\mathbb{R}), \mathscr{B}, \mu\right)$ $\equiv\left(L^{q}\right)$. The corresponding dual spaces are denoted by $\mathscr{S}_{-p}(\mathbb{R})$ and $(\mathscr{S})_{-p}$, respectively. Then we define the space $(\mathscr{S})$ of test functionals of white noise as the projective limit of the family $\left\{(\mathscr{S})_{p}, p \in \mathbb{N}_{0}\right\}$, and denote by $(\mathscr{S})^{*}$ its dual. Note that $\mathscr{S}(\mathbb{R})$ is the projective limit of the family $\left\{\mathscr{S}_{p}(\mathbb{R}), p \in \mathbb{N}_{0}\right\}$, cf. e.g. [Si 71]. Every element $\varphi \in\left(L^{2}\right)$ admits a chaos decomposition

$$
\varphi=\sum_{n=0}^{\infty} I_{n}\left(F^{(n)}\right)
$$

where $F^{(n)} \in \widehat{L^{2}\left(\mathbb{R}^{n}\right)}, \hat{\cdot}$ denoting symmetrization, and $I_{n}\left(F^{(n)}\right)$ is the multiple Wiener integral of $F^{(n)}$ of order $n$. It can be shown that $\varphi \in(\mathscr{S})$ implies

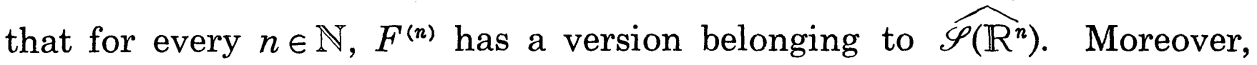
$\varphi \in\left(L^{2}\right)$ corresponding to $\left(F^{(n)} ; n \in \mathbb{N}_{0}\right)$ belongs to $(\mathscr{S})$ if and only if for every $p \in \mathbb{R}$,

$$
\|\varphi\|_{2, p}^{2}=\sum_{n=0}^{\infty} n !\left|\left(A^{\otimes n}\right)^{p} F^{(n)}\right|_{2}^{2} .
$$

The norms under the last sum will also be denoted by $\left|F^{(n)}\right|_{2, p}$.

On $\left(L^{q}\right), q>1$, and for $\xi \in \mathscr{S}(\mathbb{R})$, we define the $\mathscr{S}$-transform (cf. also [KT 80]) of an element $\varphi$ by

$$
\mathscr{S}_{\varphi(\xi)}=\int \varphi(x+\xi) d \mu(x) .
$$

Note that we have the formula

$$
\mathscr{S} \varphi(\xi)=\int \varphi(x): \exp \langle x, \xi\rangle: d \mu(x),
$$

where we have set

$$
: \exp \langle x, \xi\rangle:=\exp \left(\langle x, \xi\rangle-\frac{1}{2}|\xi|_{2}^{2}\right) .
$$

If $\varphi \in\left(L^{2}\right)$ has a chaos decomposition as in (1.1) then

$$
\mathscr{S} \varphi(\xi)=\sum_{n=0}^{\infty} \int_{\mathrm{R}^{n}} F^{(n)}(u) \xi^{\otimes n}(u) d u
$$

as an easy direct computation shows.

Since for all $\lambda \in \mathbb{C}$ and $\xi \in \mathscr{S}(\mathbb{R})$, : $\exp \lambda\langle\cdot, \xi\rangle: \in(\mathscr{P})$, we may extend the $\mathscr{S}$-transform consistently to $(\mathscr{S})^{*}$ by setting for $\Phi \in(\mathscr{S})^{*}$

$$
\mathscr{S} \Phi(\xi)=\langle\Phi,: \exp \langle\cdot, \xi\rangle:\rangle .
$$


It is not hard to see that for all $\Phi \in(\mathscr{P})^{*}$, and $\xi \in \mathscr{S}(\mathbb{R})$, the mapping $\lambda_{1} \rightarrow \mathscr{S} \Phi(\lambda \xi)$ has an entire analytic extension to $\mathbb{C}$. Clearly, it is given by

$$
z \longmapsto\langle\Phi,: \exp z\langle\cdot, \xi\rangle:\rangle, \quad z \in \mathbb{C} .
$$

In the following we shall study analytic properties of this function. To this end we introduce the following notions for mappings

$$
\begin{aligned}
f: \mathbb{C} \times \mathscr{P}(\mathbb{R}) & \longrightarrow \mathbb{C} \\
(\boldsymbol{z}, \xi) & \longmapsto f(\boldsymbol{z}, \xi) .
\end{aligned}
$$

For $R \geq 0$, and $\xi \in \mathscr{S}(\mathbb{R})$ set

$$
M(R, \xi)=\sup _{z \in \mathbf{C},|z|=R}|f(z, \xi)| .
$$

Also, we shall use the notation

$$
B_{p}^{*}=\left\{\xi \in \mathscr{S}(\mathbb{R}) ;|\xi|_{2,-p} \leq 1\right\},
$$

for real $p$.

DEFINITION 1.1.

(a) $f$ is called of $p$-order $\rho, \rho \geq 0$, if and only if

$$
\limsup _{R \rightarrow \infty} \frac{\ln \ln M(R, \xi)}{\ln R}=\rho,
$$

uniformly on $B_{p}^{*}$.

(b) Assume that $f$ is of $p$-order $\rho, \rho \geq 0 . f$ is called of $p$-type $\tau, \tau \in$ $[0,+\infty]$, if and only if

$$
\limsup _{R \rightarrow \infty} R^{-\rho} \ln M(R, \xi)=\tau,
$$

uniformly on $B_{p}^{*}$.

(c) $f$ is called of $p$-growth $(\rho, \tau)$, if and only if $f$ is of $p$-order not exceeding $\rho$, and if of $p$-order $\rho$, then $f$ is of $p$-type not exceeding $\tau$.

Assume that $F$ is a ray entire function on $\mathscr{S}(\mathbb{R})$, i.e. for every $\xi, \eta \in$ $\mathscr{S}(\mathbb{R})$, the mapping $\lambda \mapsto F(\lambda \xi+\eta)$ has an entire analytic continuation. The function $z \mapsto F(z \xi)$ is denoted by $f(z, \xi)$. Then we shall use the notions $p$-order, $p$-type and p-growth also the function $F$.

In [PS 89] a ray entire $F$ of $(-p)$-growth $(2, \tau)$, for some $p \geq 0$, and $\tau \geq 0$, was named a $U$-functional. The main result of [PS 89] was the fact that any element in $(\mathscr{S})^{*}$ has an $\mathscr{S}$-transform which is a $U$-functional, and 
conversely, for any $U$-functional $F$ there is a unique element $\Phi \in(\mathscr{S})^{*}$ with $\mathscr{S} \Phi(\xi)=F(\xi)$.

The main result of the present paper is the following theorem.

TheOREm 1.2. If $\varphi \in(\mathscr{S})$ then $\mathscr{S} \varphi$ is ray entire and for every $p \geq 0$ of p-growth $(2,0)$. Conversely, if $F$ is ray entire on $\mathscr{S}(\mathbb{R})$, and for every $p \geq 0 F$ is of p-growth $(2,0)$, then there is a unique element $\varphi \in(\mathscr{S})$ whose $\mathscr{S}$-transform is equal to $F$.

The plan of the paper is as follows. Section 2 contains the proof of Theorem 1.2. In Section 3 we discuss some of consequences and examples.

ACKNowledgement. Two of us (J.P. and L.S.) acknowledge gratefully the warm hospitality of Professor T. Hida and the Department of Mathematics at Nagoya University.

\section{§2. Proof of Theorem 1.2}

Assume that $F$ is ray entire and of $p$-order $\rho \geq 0$. If $F$ is of $p$-type 0 , we have by definition

$$
\limsup _{R \rightarrow \infty} R^{-\rho} \ln M(R, \xi)=0
$$

uniformly on $B_{p}^{*}$ and consequently

$$
\lim _{R \rightarrow \infty} R^{-\rho} \ln M(R, \xi)=0
$$

uniformly on $B_{p}^{*}$. But this implies that for every $\varepsilon>0$ we have

$$
\lim _{R \rightarrow \infty} M(R, \xi) e^{-\varepsilon R^{\rho}}=0,
$$

uniformly on $B_{p}^{*}$. On the other hand, if (2.1) holds uniformly on $B_{p}^{*}$, then it entails that $F$ is of $p$-type 0 . Thus we have proved the following result.

LEMma 2.1. $F$ is of $p$-growth $(2,0)$ if and only if for every $\varepsilon>0$ and $\xi \in \mathscr{S}(\mathbb{R})$,

$$
\lim _{R \rightarrow \infty} M(R, \xi) e^{-\varepsilon R^{2}}=0
$$

uniformly on $B_{p}^{*}$.

Now we prove the first statement of Theorem 1.2. 
Lemma 2.2. If $\varphi \in(\mathscr{S})$ then $\mathscr{S} \varphi$ is for every $p \in \mathbb{N}$ of $p$-growth $(2,0)$.

Proof. Let $\varphi \in(\mathscr{S})$ and $\xi \in \mathscr{S}(\mathbb{R})$. Denote by $z \mapsto f(z, \xi)$ the entire extension of $\lambda \mapsto \mathscr{S}_{\varphi}(\lambda \xi)$. The self-adjointness of $\Gamma(A)$ and Schwarz' inequality provide for every $q \geq 1$, and $z \in \mathbb{C}$, the following estimation.

$$
\begin{aligned}
|f(z, \xi)| & =\left|\left(\varphi,: e^{z\langle\cdot, \xi\rangle}:\right)\right| \\
& =\left|\left(\Gamma(A)^{q} \varphi, \Gamma(A)^{-q}: e^{z\langle\cdot, \xi\rangle}:\right)\right| \\
& =\left|\left(\Gamma(A)^{q} \varphi,: e^{z\left\langle\cdot, A^{-q \xi}\right\rangle}:\right)\right| \\
& \leq\|\varphi\|_{2, q}\left\|: e^{z\langle\cdot, A-q \xi\rangle}:\right\|_{2} .
\end{aligned}
$$

The last norm can easily be evaluated:

$$
\left\|: e^{z\left\langle\cdot, A^{-q \xi}\right\rangle}:\right\|_{2}=\exp \left(\frac{1}{2}|z|^{2}|\xi|_{2,-q}^{2}\right)
$$

Therefore we have the bound

$$
|f(z, \xi)| \leq\|\varphi\|_{2, q} \exp \left(\frac{1}{2}|z|^{2}|\xi|_{2,-q}^{2}\right),
$$

which is valid for all $z \in \mathbb{C}$ and all $q \in \mathbb{R}$. Clearly, this gives the estimate

$$
M(R, \xi) \leq\|\varphi\|_{2, q} \exp \left(\frac{1}{2} R^{2}|\xi|_{2,-q}^{2}\right)
$$

for all $R \geq 0$, and all real $q$.

Now choose $q$ large enough so that for given $p \in \mathbb{N}$, and given $\varepsilon>0$, $2^{2(p-q)}<\varepsilon$. Then we find for $\xi \in \mathscr{S}(\mathbb{R})$ with $|\xi|_{2,-p} \leq 1$

$$
\begin{aligned}
M(R, \xi) e^{-\varepsilon R^{2}} & \leq\|\varphi\|_{2, q} \exp \left[-\left(\varepsilon-\frac{1}{2}\left|A^{p-q} A^{-p} \xi\right|_{2}^{2}\right) R^{2}\right] \\
& \leq\|\varphi\|_{2, q} e^{-1 / 2 \varepsilon R^{2}},
\end{aligned}
$$

since for $q>p$ we have $\left\|A^{p-q}\right\| \leq 2^{p-q}$. Obviously this tends uniformly on $B_{p}^{*}$ to zero. Thus an application of Lemma 2.1 concludes the proof.

The proof of the second statement of Theorem 1.2 will be done in a sequence of lemmas.

From now on assume that $F$ is a ray entire function on $\mathscr{S}(\mathbb{R})$, which is of $p$-growth $(2,0)$. Also denote

$$
f(z, \xi)=F(z \xi), \quad z \in \mathbb{C}, \xi \in \mathscr{S}(\mathbb{R}) .
$$

The power series expansion of $f(\cdot, \xi)$ will be denoted as follows. 


$$
f(z, \xi)=\sum_{n=0}^{\infty} z^{n} f_{n}(\xi)
$$

The next result has been shown in [PS 89].

Lemma 2.3. Let $F$ be ray entire and of p-growth $(2,0)$. Then $F$ is infinitely often Gâteaux differentiable in every direction of $\mathscr{S}(\mathbb{R})$. Moreover, for all $n \in \mathbb{N}, \xi \in \mathscr{S}(\mathbb{R})$

$$
f_{n}(\xi)=\frac{1}{n !}\left(D_{\xi}^{n} F\right)(0)
$$

where $D_{\xi}$ denotes the Gâteaux derivative in direction $\xi$. In particular, $f^{(n)}(\xi)$ is homogeneous in $\xi$ of degree $n$.

Lemma 2.4. If $F$ is of $p$-growth $(2,0)$, then for all $K>0$ there is $n_{0} \in \mathbb{N}$ so that for all $n \geq n_{0}$, and for all $\xi \in \mathscr{S}(\mathbb{R})$

$$
\left|f_{n}(\xi)\right| \leq e^{-K_{n}} n !^{-1 / 2}|\xi|_{2,-p}^{n}
$$

Proof. Assume for the moment that $|\xi|_{2,-p}=1$. It follows from Lemma 2.1 that for every $\varepsilon>0$

$$
\lim _{R \rightarrow \infty} M(R, \xi) e^{-\varepsilon R^{2}}=0,
$$

uniformly on $B_{p}^{*}$.

Let $K_{1}>0$ and choose $\varepsilon=e^{-2 K_{1}}$. Choose $R_{0}$ large enough so that for all $R \geq R_{0}$

$$
M(R, \xi) e^{-\varepsilon R^{2}} \leq 1
$$

Note that $R_{0}$ is independent of $\xi \in B_{p}^{*}$. Let $R=\max \left(R_{0}, n^{1 / 2} e^{K_{1}}\right)$. Then for $n_{0}$ large enough we have $R=n^{1 / 2} e^{K_{1}}$ for all $n \geq n_{0}$. Cauchy's theorem gives the estimate

$$
\left|f_{n}(\xi)\right| \leq\left(n^{1 / 2} e^{K_{1}}\right)^{-n} e^{n},
$$

for all $n \geq n_{0}$. Stirling's theorem shows that

$$
\left|f_{n}(\xi)\right| \leq n !^{-1 / 2} e^{-\left(K_{1}-K_{2}\right) n},
$$

where $K_{2}$ is a certain constant. Choose $K_{1}$ above such that $K_{1}-K_{2}=K$ and inequality (2.2) is proved for $\xi$ with $|\xi|_{2,-p}=1$.

For general $\xi \in \mathscr{S}(\mathbb{R})$ inequality (2.2) follows now by homogeneity of $f_{n}$ 
Next we construct from $f_{n}$ by polarization an $n$-linear form $F^{(n)}$ on $\mathscr{S}(\mathbb{R})$. For $\xi_{1}, \cdots, \xi_{n} \in \mathscr{S}(\mathbb{R})$ we set

$$
F^{(n)}\left(\xi_{1}, \cdots, \xi_{n}\right)=\frac{1}{n !} \sum_{k=1}^{n}(-1)^{n-k} \sum_{l_{1}<\cdots<l_{k}} f_{n}\left(\xi_{l_{1}}+\cdots+\xi_{l_{k}}\right) .
$$

If we combine Lemma 2.4 with formula (2.3) we easily obtain the following estimate (an analogous result is proved in detail in [PS 89]).

Lemma 2.5. For every $K>0$ there is $n_{0} \in \mathbb{N}$ so that for all $n \geq n_{0}$, and all $\xi_{1}, \cdots, \xi_{n} \in \mathscr{S}(\mathbb{R})$

$$
\left|F^{(n)}\left(\xi_{1}, \cdots, \xi_{n}\right)\right| \leq e^{-K n} n !^{-1 / 2} \prod_{k=1}^{n}\left|\xi_{k}\right|_{2,-p} .
$$

Let $\mathscr{S}_{-p}\left(\mathbb{R}^{n}\right)$ denote the completion of $\mathscr{S}\left(\mathbb{R}^{n}\right)$ under the norm $|g|_{2,-p}$ $\equiv\left|\left(A^{\otimes n}\right)^{-p} g\right|_{2}$. Note that the set of elements $\left\{\bigotimes_{k=1}^{n} \xi_{k} ; \xi_{k} \in \mathscr{P}(\mathbb{R})\right\}$ is total in $\mathscr{S}_{-p}\left(\mathbb{R}^{n}\right)$. Then inequality (2.4) tells us that the $n$-linear form $F^{(n)}$, which we may consider as a linear form on $\mathscr{S}(\mathbb{R})^{\otimes n}$, has a continuous extension to $\mathscr{S}_{-p}\left(\mathbb{R}^{n}\right)$. We denote this extension by the same symbol. Therefore, we may now consider $F^{(n)}$ as an element in $\mathscr{S}_{p}\left(\mathbb{R}^{n}\right)$, the dual of $\mathscr{S}_{-p}\left(\mathbb{R}^{n}\right)$. The next lemma estimates the $|\cdot|_{2, p}$-norm of $F^{(n)}$ under a slightly stronger hypothesis.

LEMMA 2.6. Assume that $F$ is of $(p+q)$-growth $(2,0)$ with $q>1 / 2$. Then for all $K>0$ there is $n_{0} \in \mathbb{N}$ so that $n \geq n_{0}$ implies

$$
\left|F^{(n)}\right|_{2, p} \leq n !^{-1 / 2} e^{-K n} \text {. }
$$

Proof. Note that

$$
\left|F^{(n)}\right|_{2, p}^{2}=\sum_{k_{1}, \cdots, k_{n}=0}^{\infty}\left|F^{(n)}\left(A^{p} e_{k_{1}}, \cdots, A^{p} e_{k_{n}}\right)\right|^{2},
$$

where $\left\{e_{k} ; k \in \mathbb{N}_{0}\right\}$ is any CONS of $L^{2}(\mathbb{R})$ in the domain of $A^{p}$. Choose $e_{k}$ to be the $k$-th Hermite function and note that $A e_{k}=2(k+1) e_{k}, k \in \mathbb{N}_{0}$. Then we know that for any $K_{1}>0$ there exists $n_{0}$ (depending on $K_{1}, p, q$ ) so that for all $n \geq n_{0}$ we have

$$
\begin{aligned}
\left|F^{(n)}\left(A^{p} e_{k_{1}}, \cdots, A^{p} e_{k_{n}}\right)\right| & \leq n !^{-1 / 2} e^{-K_{1} n} \prod_{l=1}^{n}\left|A^{p} e_{k_{l}}\right|_{2,-(p+q)} \\
& =n !^{-1 / 2} e^{-K_{1} n} \prod_{l=1}^{n}\left(2\left(k_{l}+1\right)\right)^{-q} .
\end{aligned}
$$

Therefore, 


$$
\begin{aligned}
& \sum_{k_{1}, \cdots, k_{n}=0}^{\infty}\left|F^{(n)}\left(A^{p} e_{k_{1}}, \cdots, A^{p} e_{k_{n}}\right)\right|^{2} \leq n !^{-1} e^{-2 K_{1} n} 2^{-2 q n}\left(\sum_{k=0}^{\infty}(k+1)^{-2 q}\right)^{n} \\
& \leq n !^{-1} e^{-2\left(K_{1}-K_{2}\right) n},
\end{aligned}
$$

for some constant $K_{2}>0$. Finally, choose $K_{1}$ so that $K_{1}-K_{2}=K$.

LEMMA 2.7. If $F$ is of $(p+q)$-growth $(2,0)$ for some $q>1 / 2$, then there is a unique element $\varphi$ in $(\mathscr{S})_{p}$ so that $\mathscr{S}_{\varphi}=F$.

Proof. First of all note that the hypothesis that $F$ is of $(p+q)$ growth $(2,0)$ implies that for every $n \in \mathbb{N}, F^{(n)} \in \mathscr{S}_{p+q}\left(\mathbb{R}^{n}\right)$, so in particular $F^{(n)} \in \mathscr{S}_{p}\left(\mathbb{R}^{n}\right)$. Also, by construction $F^{(n)}$ is symmetric. Now set

$$
\varphi=\sum_{n=0}^{\infty} I_{n}\left(F^{(n)}\right) .
$$

By construction, $\varphi \in(\mathscr{S})_{p}$ if and only if (1.2) is finite. But the estimate of Lemma 2.6 shows that this is true.

It is clear that the second statement of Theorem 1.2 follows immediately from Lemma 2.7 .

\section{§ 3. Consequences and examples}

As a first illustration of our theorem let us investigate the question whether the composition of $f \circ X_{\eta}$ of a function $f$ in the Schwartz space $\mathscr{S}(\mathbb{R})$ of test functions with the random variable $X_{\eta}=\langle\cdot, \eta\rangle, \eta \in \mathscr{S}(\mathbb{R})$, belongs to $(\mathscr{S})$. As a special case, let us choose $f$ to be the Gauss function $f(u)=\exp \left(-\frac{1}{2} u^{2}\right)$. It is an easy exercise to compute

$$
\mathscr{S} \varphi(\xi)=\left(1+|\eta|_{2}^{2}\right)^{-1 / 2}-\exp \left(-\frac{1}{2} \frac{(\eta, \xi)^{2}}{1+|\eta|_{2}^{2}}\right)
$$

for

$$
\varphi(x)=f \circ X_{\eta}=\exp \left(-\frac{1}{2}\langle x, \eta\rangle^{2}\right) .
$$

It is obvious that $\mathscr{S} \varphi$ is not of type 0 . Therefore $\varphi$ does not belong to $(\mathscr{S})$, and consequently in general the answer to the above raised question is negative.

Based on Theorem 1.2 we can very easily establish a result which has already been proved in [PR 89] by other methods, and which describes a large class of examples in $(\mathscr{S})$ of the above type. 
TheOREM 3.1. Let $\eta \in \mathscr{S}(\mathbb{R})$, and $f$ be a function on $\mathbb{R}$ whose Fourier transform $\hat{f}$ belongs to $L^{1}(\mathbb{R})$ and has compact support. Then $f \circ X_{\eta} \in(\mathscr{S})$.

Proof. By Fubini's theorem, we find that

$$
\mathscr{S} f \circ X_{\eta}(\xi)=(2 \pi)^{-1 / 2} \int_{\mathrm{R}} \hat{f}(p) \mathscr{S} e^{i p\langle\cdot \eta \eta}(\xi) d p .
$$

It is plain to compute the $\mathscr{S}$-transform under the last integral:

$$
\mathscr{S} e^{i p \prec \cdot \eta \eta}(\xi)=\exp \left(i p(\xi, \eta)-\frac{1}{2} p^{2}|\eta|_{2}^{2}\right),
$$

where $(\cdot, \cdot)$ is the inner product of $L^{2}(\mathbb{R})$. Thus we have the formula $(z \in \mathbb{C})$

$$
\mathscr{S} f \circ X_{\eta}(z \xi)=(2 \pi)^{-1 / 2} \int_{\mathrm{R}} \hat{f}(p) \exp \left(i z p(\xi, \eta)-\frac{1}{2} p^{2}|\eta|_{2}^{2}\right) d p .
$$

First of all note that the fact that $\hat{f}$ has compact support allows us to differentiate with respect to $z \in \mathbb{C}$ under the integral sign in (3.1). From this we obtain immediately that the function $z \mapsto \mathscr{S} f \circ X_{\eta}(z \xi)$ admits the Cauchy-Riemann equations and therefore $\mathscr{S} f \circ X_{\eta}$ is ray entire.

Assume that supp $\hat{f} \subset[-N, N], N>0$. Then we can estimate for every $p \in \mathbb{N}$ as follows.

$$
\begin{aligned}
M(R, \xi) & \equiv \sup _{|z|=R}\left|\mathscr{S} f \circ X_{\eta}(z \xi)\right| \\
& \leq(2 \pi)^{-1 / 2}|\hat{f}| e^{R N|(\xi, \eta)|} \\
& \leq(2 \pi)^{-1 / 2}|\hat{f}|_{1} e^{R .\left.|\xi|\right|_{2,-p|\eta|}, p},
\end{aligned}
$$

where $|\cdot|_{1}$ is the $L^{1}(\mathbb{R})$-norm. Clearly, this bound shows that $\mathscr{S} \circ \circ X_{\eta}$ is for every $p \in \mathbb{N}$ of $p$-order 1 and consequently of $p$-growth $(2,0)$.

Example 3.2. Note that the function $f(u)=u^{-1} \sin u, u \in \mathbb{R}$ satisfies the hypothesis of Theorem 3.1. Therefore $\varphi$ given by

$$
\varphi(x)=\frac{\sin \langle x, \eta\rangle}{\langle x, \eta\rangle}, \quad x \in \mathscr{S}^{\prime}(\mathbb{R}), \eta \in \mathscr{S}(\mathbb{R}),
$$

belongs to $(\mathscr{S})$. Since $(\mathscr{S})$ is an algebra (e.g. [HPS 88]), so $\varphi^{2} \in(\mathscr{S}) . \varphi^{2}$ was used in [PR 89] to show that every smooth bounded cyclinder function can be approximated by sequences in $(\mathscr{S})$ in the topology defined by certain Dirichlet forms over $\mathscr{S}^{\prime}(\mathbb{R})$.

Example 3.3. If $f$ is entire and of growth (2,0) (cf. e.g. [Bo 54]), and 
we pick $\eta \in \mathscr{S}(\mathbb{R})$, then for all $p \in \mathbb{N}$,

$$
F(\xi):=f \circ(\eta, \xi)
$$

is of $p$-growth $(2,0)$. This follows as above from the estimate $|(\eta, \xi)| \leq$ $|\eta|_{2, p}|\xi|_{2,-p}$, and the finiteness of $|\eta|_{2, p}$ for all $p$. Therefore any such pair $(f, \eta)$ gives rise to an element in $(\mathscr{S})$. For example, we may choose for $f$ the functions $z \mapsto \cos \sqrt{\bar{z}}$ or $z \mapsto \Gamma(z)^{-1}$.

Note added in proof. After this manuscript was submitted, we learned that Ju. G. Kondrat'ev had obtained similar results in "Nuclear spaces of entire functions in problems of infinite-dimensional analysis"; Soviet Math. Dokl., 22 (1980), 588-592.

\section{REFERENCES}

[Bo 54] Boas, R. P., Entire Functions, New York, Academic Press (1954).

[HKPS] Hida, T., Kuo, H.-H., Potthoff, J. and Streit, L., White Noise: An Infinite Dimensional Calculus, Monograph in preparation.

[HPS 88] Hida, T., Potthoff, J. and Streit, L., Dirichlet forms and white noise analysis, Commun. Math. Phys., 116 (1988), 235-245.

[KT 80] Kubo, I. and Takenaka, S., Calculus on Gaussian white noise I, Proc. Japan Acad., 56A (1980), 376-380.

[PR 89] Potthoff, J. and Röckner, M, On the contraction property of energy forms in infinite dimensions, BiBoS Preprint (1989), to appear in J. Funct. Anal.

[PS 89] Potthoff, J. and Streit, L., A characterization of Hida distributions, BiBoS Preprint, no. 406 (1989).

[Si 71] Simon, B., Distributions and their Hermite expansions; J. Math. Physics, 12 (1971), 140-148.

H.-H. Kuo

J. Potthoff

Department of Mathematics

Louisiana State University

Baton Rouge, LA 70803

U.S.A.

L. Streit

BiBoS

Universität Bielefeld

Bielefeld

Germany

and

Area de Matemática

Universidade do Minho

Braga

Portugal 